\title{
Italique
}

Poésie italienne de la Renaissance

VII | 2004

Varia

\section{Pétrarque au partage de midi}

\section{Francisco Rico}

\section{OpenEdition}

\section{Journals}

Édition électronique

URL : http://journals.openedition.org/italique/124

DOI : 10.4000/italique.124

ISSN : 1663-4438

\section{Éditeur}

Librairie Droz

\section{Édition imprimée}

Date de publication : 1 novembre 2004

Pagination : 9-26

ISBN : 2-600-00976-0

ISSN : 1423-3983

Référence électronique

Francisco Rico, « Pétrarque au partage de midi », Italique [En ligne], VII | 2004, mis en ligne le 05 octobre 2009, consulté le 20 avril 2019. URL : http://journals.openedition.org/italique/124 ; DOI : 10.4000/italique.124 
F R A N C I S C O R I C O

PÉTR A R Q U E

A U PARTAGE DE MIDI 


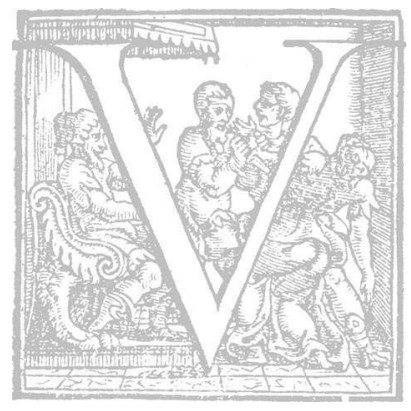

Ne partie immense de la poésie lyrique européenne postérieure à François Pétrarque a consisté pour les uns à l'imiter et pour d'autres à s'en démarquer, mais Pétrarque, déjà, avait aspiré à se démarquer de lui-même. Le Canzoniere s'ouvre et s'achève en effet sur des palinodies. Au terme du recueil, la chanson à la Vierge déplore "quante lagrime», "quante lusinghe et quanti preghi» remplissent le livre «indarno", pour la "pena» et le "grave danno» du poìte avenglé, tenaillé dans son âme par la beauté d'une "poca mortal terra caduca ». Au commencement, Voi ch'ascoltate présente comme un "giovenile errore 》 toutes les attitudes et tous les sentiments, "le vane speranze e 'l van dolore » inspirant la plupart des "rime sparse » qui font suite. Mais l'on ne peut comprendre correctement le langage du sonnet prologal des Rerum vulgarium fragmenta en considérant uniquement son sens littéral dans l'usage quotidien.

En réalité, dans Voi ch'ascoltate comme en bien des endroits de l'cuvre de Pétrarque - qu'il s'agisse de prose ou de vers, de latin ou de langue vulgaire -, "errore» constitue à peu de choses près un terme technique emprunté à la tradition stö̈cienne pour désigner la falsa opinio qui trouble la vision $d u$ commun des mortels, alimente les "speranze ", le "dolore» et les autres affectus ou perturbationes animi, provoque la désagrégation de l'esprit en "pensieri » aussi « sparsi » que les rimes venant les refléter. De toute évidence, Pétrarque apparaît ici comme un "altr' nom»: il n'est plus ce qu'il était autrefois, il arbore désormais l'air grave du sage stö̈cien et n'bésite pas à dénoncer les poèmes $d u$ Canzoniere comme autant de rerum vulgarium fragmenta dans leur fond et dans leur forme, comme de vulgaires morceaux dignes $d u$ "popol» ignorant.

Il ne s'agit pas ici d'une simple fiction plus ou moins placée sous le signe des précédents bien connus offerts par la littérature latine et les troubadours: à plusieurs reprises, lillustre Italien dont nous commémorons le septième centenaire a voulu se convertir en " altr' nom » et y est parvenu. Contre la vieille idée d'un Pétrarque "senza storia», nous avons aujourd'bui la certitude que son itinéraire intellectuel, littéraire et bumain, bien loin d'être rectiligne, a été marqué par un grand nombre d'bésitations, de rectifications et même de changements sensibles d'orientation. J'aimerais maintenant attirer un instant votre attention sur un des moments décisifs de cette trajectoire passionnée et émouvante: ce moment du partage de midi où, à la croisée des chemins, déjà à l'âge mûr, Pétrarque résout les incertitudes qui l'avaient hanté durant ses longues années de formation et s'engage d'un pas ferme sur la voie qui le 
conduira à son plein épanouissement, à la fois en tant qu'écrivain et en tant qu'bomme.

Dirigeons pour commencer notre regard sur un printemps romain. En avril I34I, Pétrarque entrait dans la salle d'audiences du Capitole, afin d'y recevoir la couronne de laurier pour laquelle il avait tant lutté et noué tant d'intrigues. Il avait alors trente-sept ans (selon sa façon de compter) et, hormis la poésie en langue vulgaire, l'extension de son cuvre était encore assez. limitée. Avec les données qu'il avait à sa portée et avec un certain optimisme, E. H. Wilkins calculait que l'écrivain avait rédigé alors moins de mille cinq cents vers latins. Ils ne devaient pas être beaucoup plus nombreux. En I336, Rinaldo Cavalchini da Villafranca mentionne deux seuls échantillons de la muse latine de Pétrarque, "dans lesquelles il déplore la situation de l'Italie et demande en larmes le retour de son époux à Rome» ("status Ytalie cum deploratur et Urbi / adventum sponsi frons lacrimosa petit»). Nous connaissons depuis toujours ces deux pièces, la seconde et la troisième du premier livre des Epystole appelées metriche. Rinaldo le supplie, s'il compose « quid ... novitatis », de ne pas manquer de le lui envoyer. En 1336, le corpus de Pétrarque est presque identique à celui que nous pouvons reconstruire de nos jours. Cinq ans après, alors que notre humaniste gravissait les marches du Capitole, l'inventaire s'était accru, surtout avec les deux grandioses projets que sont l'Africa et le De viris illustribus, un poème épique et une collection d'ébauches historiques. Grandioses, certes, mais, en définitive, uniquement des projets. Le laurier couronnait de belles espérances, plutôt que des réalités effectives.

Durant le printemps 1353, lors de sa première visite à la basilique de Sant'Ambrogio, si proche de sa demeure milanaise, Francesco n'avait pas l'intention, ou du moins n'envisageait pas pour l'immédiat, de consacrer le temps et la tranquillité dont il disposait à terminer l'Africa et le De viris : le gigantesque De remediis utriusque fortune était l'cuvre majeure qu'il méditait et qu'il se disposait à accomplir, tandis qu'il s'occupait de la révision et de la suite de son recueil de lettres en prose. Depuis qu'au début de l'année I345 Pétrarque avait abandonné la rédaction des Rerum memorandarum, les livres qu'il avait pratiquement achevés étaient plutôt dans l'esprit du De remediis et des Familiares que dans celui des projets couronnés en I34I: il s'agissait surtout du De vita solitaria, le long panégyrique de la solitude occupée "par l'amour des lettres et la vertu»; du De otio religioso, à la louange de la vie monacale et de la contemplation, et du De secreto conflictu curarum mearum, où il se demande entre autres s'il y a lieu de continuer à travailler à l'Africa et au De viris.

Il est vrai qu'entre 1346 et 1348 , il avait achevé la partie la plus importante du Bucolicum carmen, mais il est également vrai que dans les Invective 
contra medicum Pétrarque, sans nier le fait qu'autrefois, encore jeune, "olim, iuveniliter », il avait ambitionné le titre de poète, déclare qu'en cette même année 1353, cela faisait déjà sept ans qu'il ne lisait plus de poésie. En effet, il ne semble pas bon de "vieillir en faisant les mêmes études que celles auxquelles on s'est consacré adolescent» ("in eisdem studiis agere senectutem in quibus adolescentia acta est »). La « maturitas», ajoute-t-il, l'a mené vers d'autres centres d'intérêt. Que fait-il donc? "Melior fieri studeo, si possim » ("j'essaie d'être meilleur, si je peux y parvenir»), en implorant l'aide de Dieu et en me complaisant «in sacris literis» (" aux saintes lettres»). Que fait-il encore? il lutte pour rectifier "les erreurs du passé» ("preteriti temporis errata »). Et encore ceci: "je ne lis pas les poètes, mais j’écris des choses que pourront lire ceux qui viendront après moi [...], en me contentant de l'approbation de certains» ("Non poetas lego, sed scribo quod legant qui post me nascentur [...], raro plausore contentus »). En fin de compte, ce qui importe avant tout, pour lui, c'est d'apprendre à devenir un homme mûr, " maturescere ». En quoi il fait tout le contraire de son concurrent, le médecin du Pape: " comme le vieil homme qui apprend l'alphabet, et dont Sénèque se moque, tu passes tes vieux jours dans les lieux mêmes où tu vécus ton enfance» ("elementarius senex irrisus a Seneca, ibi senectutem agis, ubi pueritiam exegisti »), c'est-à-dire parmi des enfantillages aussi prétentieux que vains.

Le Privilegium du Couronnement le célébrait comme « magnum poetam et historicum ». Mais à présent, il réclame le titre de "philosophus», et par "philosophe ", il entend celui dont la mission consiste à "replier son âme sur soi », " reflectendum ad se animum », et qui, en devenant meilleur, rend bon autrui, "bonum facere auditorem ac lectorem ». Les recueils de lettres, le Secretum, les opuscules polémiques, l'immense vade-mecum de l'bumaniste chrétien que représente le De remediis obéissent à la volonté qu'il a d'accomplir cette mission.

C'est là sans aucun doute le trait dominant de la «maturitas» de Pétrarque: l'évolution qui fait que le "poeta et historicus " couronné à Rome devient le "philosophus» que nous retrouvons à Milan. Naturellement, je n'essaie pas de ressusciter la vieille légende, entretenue d'ailleurs par l'bumaniste lui-même, mais définitivement démentie par Giuseppe Billanovich, selon laquelle Pétrarque n'aurait même pas lu la Bible jusqu'à l'époque du De otio, et selon laquelle ce n'est qu'alors que l'écrivain aurait découvert les lettres chrétiennes et s'y serait consacré avec la ferveur d'un converti. Au contraire, j'ai déjà expliqué à différentes occasions que le passage à la "philosophia impliquait cher. notre écrivain la réconciliation d'aspects de sa personnalité et de son expérience intellectuelle et littéraire qui lui étaient propres dès ses premières lectures, mais qu'il ne parvint à concilier de manière satisfaisante qu'une fois passé la quarantaine. 
C'est que le classicisme à outrance de son cuure de jeunesse répondait sans aucun doute à une facette fondamentale de Pétrarque, mais était loin d'en être l'expression achevée. Pour nous limiter à un seul exemple, essentiel, il suffira de faire remarquer que Pétrarque, malgré toutes ses faiblesses, était un chrétien d'une orthodoxie et d'une piété inébranlables. Mais dans les grandes cuvres auxquelles il rêva durant sa jeunesse - surtout dans l'Africa et dans le De viris - l'idéal de l'imitatio ne pouvait, sous peine de se trabir, laisser libre cours au sentiment religieux. Ainsi, dans le prologue de l'épopée, Pétrarque n'ose se référer au Christ qu'en utilisant le genre de périphrases qu'Érasme caricaturera plus tard dans le Ciceronianus, et à l'aide de circonlocutions comme «certissima mundi spes superumque decus»; et c'est pour cette même raison qu'il souligne, à la fin du De vita solitaria, combien il lui a été agréable, cette fois, de nommer fréquemment le Christ, en s'éloignant du «mos veterum ", le style des anciens. De ce silence artificiel, dans sa jeunesse, ne pouvaient naître qu'un certain malaise, un sentiment de frustration, de manque de plénitude dans l'expression littéraire.

Cependant, la solution était bien simple, car Pétrarque avait toujours fondé son occupation de letterato sur la conviction que l'Antiquité gréco-latine illustrait la convergence ultime de la vérité révélée et de la loi naturelle, l'accord entre la morale chrétienne et les qualités éthiques permanentes qui, par la volonté du Créateur, définiraient la condition bumaine: ce "quid bumanum omniumque gentium comune », "ce quelque chose de propre à l'être bumain et de commun à tous », qu'il essaya de mettre dans la bouche du Magon agonisant, et que Dieu, il en était convaincu, avait concédé, au plus haut degré, aux grands auteurs classiques.

Il suffisait, par conséquent, de rendre explicite cette conviction, que Francesco avait toujours eue, et d'adapter en littérature les données définies et conciliées en esprit depuis l'adolescence, en soulignant la concordance entre classicisme et christianisme. Par ailleurs, telle qu'elle est conçue par Pétrarque, la "philosophia » ne peut atteindre son objectif sans recourir aux instruments de l'" eloquentia» antique: seules les lettres latines constituent la légitime "racine de toutes nos habiletés, de tout l'art de notre discipline et le fondement de tous les savoirs» ("radix artium nostrarum et omnis scientie fundamentum»), et seule la rhétorique parvient à atteindre la persuasion proprement dite, celle qui touche les sentiments et les comportements.

Mais je n'ai pas l'intention de m'étendre aujourd'bui sur cet aspect capital de la maturité de Pétrarque. Je préfere esquisser d'autres caractéristiques qui accompagnent le développement de l'bumaniste en tant que "philosophus» et qui, en contribuant fortement à caractériser sa production de l'âge mûr, sont tout à fait accessibles quand on les contemple d'un point de vue biographique: non seulement, donc, en adoptant une approche littéraire et culturelle à valeur 
générique, ni même seulement à la lumière des circonstances et des idées sociales et politiques, mais également, et sans dédaigner aucune de ces approches, du point de vue plus proprement personnel, en adoptant la perspective singulière et individuelle d'un bomme qui a sa propre histoire et a atteint, dans sa vie, un moment particulier.

Les caractéristiques sur lesquelles je m'arrêterai sont au nombre de trois, toutes trois solidaires et inséparables de la conversion de Pétrarque de "poeta » en "philosophus». En effet, au fur et à mesure que dans son cuvre l'attitude du "philosophus» gagne du terrain, d'une part les éléments subjectifs gagnent de l'importance, et d'autre part l'bumaniste cherche à atteindre un plus vaste public, tandis que sa production, forte d'une extraordinaire cobérence d'esprit, maintient dans sa forme un caractère pour le moins fragmentaire.

Quand on compare les textes antérieurs et ceux immédiatement postérieurs à I345, même sans étendre la perspective aux travaux plus anciens, les différences sautent aux yeux. Dans le De viris, dans l'Africa, dans les Rerum memorandarum, c'est le récit objectif centré sur les sujets classiques qui domine. Dans le De vita, dans le De otio et le Secretum, c'est l'accent subjectif et les réflexions éthiques teintées de spiritualité explicite qui l'emportent. Et dorénavant ce sera là la dimension principale du corpus pétrarquiste: son cuvre se centrera désormais sur la première personne, sur l'expérience individuelle en tant que chemin menant à l'analyse morale de toutes choses, elle se fondera sur l'exploration et la connaissance du "je» comme méthode et but.

En fait, celui qui connaît quelque peu la personnalité de notre humaniste ne peut qu'être surpris du fait qu'il ait décidé de choisir le modèle de l'épopée et du récit historique pour son grand cuvre littéraire d'avant quarante ans. Il est vrai que le genre épique se présentait comme le sommet de l'accomplissement poétique et il est également vrai que l'historiographie était le terrain le plus apte à exhiber l'incomparable érudition classique de l'auteur. Mais on peut difficilement imaginer des genres littéraires plus éloignés du génie de Pétrarque. Depuis Umberto Bosco, on sait que le sens de la fugacité et de la caducité de la vie, "il senso della labilità », domine une partie très importante de l'xuvre en prose et en vers de Pétrarque, tout particulièrement sa poésie en langue vulgaire. Quand on rapproche ce grand thème du Canzoniere, des Epystole et de tant d'autres pages, de l'obsession chronologique dont témoignent à chaque instant ses manuscrits et ses cabiers, on comprend qu'il y avait chez. Pétrarque la volonté irrépressible, et angoissée, de saisir et traduire en écriture chaque instant de son existence, afin de pouvoir les fixer, afin de les avoir en permanence à sa portée et de pouvoir en faire les pièces d'une histoire unitaire, d'un ensemble saisissable en tant que tel. Nous trouvons cependant peu de traces de cet élan congénital avant que l'bumaniste ne fête ses quarante ans: 
hormis les Rerum vulgarium fragmenta, qui ont toujours leur propre spécificité, à peine quelques lettres, en prose ou en vers, sans liens entre elles, et dont on ignore quand elles furent écrites dans la version qui nous est parvenue. Il était donc presque inévitable, semble-t-il, que notre bumaniste s'engage sur la voie qui le conduirait vers des genres et des manières plus largement ouverts à la subjectivité que ne le sont la poésie épique et l'bistoriographie. Les diverses modalités d'écriture autobiographique - avec en premier lieu la lettre - et d'expression personnalisée - qui va du dialogue à ce que nous appellerions aujourd'bui l'essai - satisfaisaient son besoin inné de prendre acte des moindres événements quotidiens, pour les saisir et leur donner forme, et rendaient ainsi possible l'intégration de sa dimension humaine particulièrement complexe et de ses tâches littéraires. La "philosophia » qu'il avait embrassée en variant les objectifs, mais non pas les fondements les mieux ancrés de son cuvre antérieure à 1345 , l'exhortait à s'adonner à la connaissance de soi et à ne pas séparer de sa propre personne les enseignements qu'il pouvait offrir à autrui. L'on comprend, dès lors, que ses xuvres de l'âge mûr débordent de subjectivité, qu'elles comportent une forte charge autobiographique et qu'une grande partie de tout ce qui a trait à lui-même ait la portée d'un manifeste et se donne comme visée de décrire une trajectoire paradigmatique qui irait, pour ainsi dire, de la philologie à la philosophie et où le portrait suggestif d'un individu soit à la fois une proposition éthique et un programme culturel.

N'oublions pas non plus qu'il devait probablement se sentir obligé de rendre compte de cet itinéraire, de donner une explication personnalisée du cheminement qui l'avait converti en "philosophus». Durant plusieurs années, usant de la coquetterie qui caractérise le spécialiste qui feint de s'intéresser uniquement aux sujets qui font partie de son domaine d'étude et ne prête attention à aucun autre problème, tels les aristotéliciens radicaux qu'il attaquera ensuite avec force dans le De ignorantia, il s'était plu à se présenter uniquement comme connaisseur du monde classique. La fausse pédanterie est bien connue avec laquelle il déclare dans les Rerum memorandarum qu'il va traiter exclusivement de sujets tirés " ex secularibus literis 》, "des lettres profanes", parce qu'il est conscient du fait qu'il ignore les lettres sacrées, "ignorantie aliarum conscius », et qu'il n'est pas capable de s'écarter des études profanes, " a secularibus studiis». Il ne pouvait donc pas devenir "philosophus»du jour au lendemain, ni se présenter comme tel sans se justifier aussi bien au plan théorique que personnellement, au plan biographique. L'cuvre de l'âge mûr devait être à plusieurs égards, si ce n'est une palinodie, du moins apologia pro vita sua.

La rhétorique, art public et pratique, exige la prise en compte des coordonnées spécifiques non seulement du destinataire, mais également de l'orateur ou du philosophe qui la met à profit. Les lettres à Cicéron montrent excellemment à 
quel point Pétrarque était profondément fâché de la disparité « inter linguam et animum, inter doctrinam et vitam ", " entre la langue et l'âme, entre la théorie et la pratique », et peiné du contraste entre « mores et verba », tel qu'il le découvrait en particulier chez les épigones de la scholastique. Mais ce n'est pas seulement pour des raisons de procédure et de cohérence doctrinale qu'il lui importait de soigner l'image que la postérité et surtout ses contemporains devaient se faire de lui : cette image était aussi son capital le plus précieux.

A l'époque du Couronnement, aussi longtemps qu'il vécut sous la protection des Colonna, sa culture exceptionnelle fut bien évidemment appréciée et utilisée par ses protecteurs, mais, en fin de compte, il n'était pas là et ne restait pas à leur service en qualité de "poeta et historicus». Lorsqu'il voulut s'éloigner de la curie papale et du milieu des cardinaux, ce qu'il pouvait vendre - que l'on me pardonne la dureté de l'expression que j'emploie, par souci de brièveté -, ce qu'il voulait lancer sur le marché des puissants, ce n'était pas la fonction et l'image d'un assistant plus ou moins distingué, paré d'une plus ou moins grande érudition, mais plutôt l'image même du "philosophus", l'image du conseiller des princes, du sage et du savant capable de rapporter les nécessités de la politique et de la vie pratique aux raisons de la culture la plus nouvelle et la plus haute, l'image d'un personnage dont la seule présence auprès d'un seigneur donnait à celui-ci une respectabilité et un air de solidité intellectuelle. Il est possible qu'il ait encore proposé ses services au Roi Robert d'une part comme "poeta et historicus", et d'autre part également comme collaborateur. Mais vers 1349, et, par exemple, devant Jacopo da Carrara, dont il pleura l'assassinat " avec l'émotion d'un homme plutôt que celle d'un philosophe », "bumani potius affectus quam philosophici», l'bumaniste se présentait sans doute déjà " en bloc », sous la seule figure du "philosophus».

Pétrarque se savait l'objet de la curiosité de nombreuses personnes ("Iam noscimur, legimur, indicamur, iamque hominum voces evadendi celandique ingenium nulla spes»), et il lui importait d'entretenir, même au prix de certaines distorsions, une image attrayante de soi: une image non nécessairement exemplaire, loin de là, mais qui devait être riche, complexe, stimulante et vive. Il avait, par ailleurs, trop baigné dans la littérature pour qu'il ne finisse pas par construire lui-même son propre personnage. Or, l'écriture lui offrait l'occasion de devenir quelqu'un d'autre, et ce, sans pour autant cesser d'être lui-même, sans renoncer à assumer toutes les identités voulues, sans renoncer à développer au moyen du langage des possibilités non réalisées ou qui, autrement, seraient sans intérêt. Mais la construction de cette image toute en demi-teintes, pleine d'ambiguïtés, de wishful thinking, cette image rendue par un art littéraire infaillible, à laquelle il s'applique surtout dans les Familiares et dans le Secretum, composés aux environs de 1350, était également de convenance disons "commerciale 》: 
de convenance pour annoncer lui-même le "produit» qu'il lançait sur le marché.

C'est toujours la volonté de s'en tenir aux convenances qui explique pour une large part les limites des autobiographies pétrarquistes. Les lettres Ad Atticum l'ont poussé à compiler les Familiares, mais elles n'en furent en aucune façon le modèle. Pétrarque ne pouvait présenter la réalité de son existence avec le naturel et la simplicité solide de Cicéron, et il devait succomber au charme de Sénèque (et peut-être même de Pierre de Blois, qui lui ressemble tant); il devait également succomber aux généralisations, à l'attitude moralisatrice, aux exempla, à la littérature. Les Confessiones sont à l'origine du Secretum, mais ne sont pas pour autant le modèle du Secretum. Contrairement à saint Augustin, Francesco ne parlait pas à Dieu et ne se parlait pas à lui-même non plus. Il réservait à Dieu la prière et la pénitence, le sacrement que Magon devinait et prophétisait. Pour sa part, sa prodigieuse mémoire et les indéchiffrables annotations des mémoriaux intimes à la fin du manuscrit des lettres d'Abélard et d'Héloïse lui suffisaient. Il s'adressait, quant à lui, à un public d'admirateurs et d'éventuels mécènes, auxquels il s'efforçait d'expliquer le nouveau rôle de "philosophus» avec lequel il entrait en scène, après tant de paganisme ornemental, après tant de « sospiri » et de «vane speranze » dus à Laura. Je ne doute pas que si à cette époque Pétrarque s'oriente vers la "philosophia ", et relègue la poésie à une seconde place, c'est que cette nouvelle orientation est le résultat d'une démarche spirituelle profonde et pleinement sincère. Mais je soulignerai également que pendant ces années-là (la quarantaine), cette réorientation présentait pour lui un intérêt tout particulier, puisqu'elle devait lui permettre d'atteindre le modus vivendi qui allait être celui de sa vieillesse.

L'une des tâches qui semblait convenir le mieux, dans cette perspective, consistait à déployer son savoir vers d'autres horizons. L'Africa et le De viris ne répondaient pas à son ambitieux projet originaire et seule une faible minorité pouvait apprécier ces textes. On comprend, dès lors, que Pétrarque, comme tout spécialiste qui croit en la valeur de sa discipline, ait senti le désir et la tentation de cristalliser son érudition au travers de formes et de sujets plus amplement accessibles et plus directement liés à la réalité du moment; on comprend également pourquoi il ressentait une profonde satisfaction à l'idée de devenir l'apôtre par excellence et l'incarnation vivante des studia humanitatis, le maître dont le prestige et le succès prowvaient que, dans la société de l'époque, il pouvait y avoir une place importante pour la nouvelle culture. Comme je viens de le rappeler, jamais Pétrarque n'a douté, même quand il ne le disait pas explicitement, de la transcendance morale et religieuse des études classiques. Il lui suffisait, par conséquent, d'appliquer cette certitude à des domaines d'intérêt plus général, lui permettant de rendre la culture antique 
plus accessible: il fallait parvenir à démontrer que dans la vie quotidienne, dans les relations d'amitié, dans les avatars de la politique, dans les problèmes individuels ou sociaux, l'béritage antique représentait la culture bumaine la plus appropriée pour approfondir ce que la religion enseignait.

Tels étaient, et tels sont peut-être encore actuellement, la leçon suprême, le message ultime de Pétrarque. A partir de la décennie qui s'étend entre la quarantaine et la cinquantaine, il laisse de côté les fruits de son activité réservés jusque-là à une petite élite et il s'applique à illustrer ces idéaux à travers une série de textes qui impliquent un important changement d'orientation. Il s'agit désormais non pas tant d'écrits occasionnels ou de circonstance que d'écrits qui s'adressent à des destinataires concrets, qui concernent des situations spécifiques ou des questions d'actualité; et le modus procedendi de Pétrarque, sa méthode, consiste dès lors essentiellement à éclairer chaque thème en recourant aux lectures classiques pour démontrer qu'elles sont toujours actuelles et valables, toujours profitables et susceptibles d'être traduites " in actum », ainsi que d'être appliquées « ad vitam ».

L'auvre de Pétrarque, à l'âge mûr, et par-delà jusqu'au soir de sa vie, est donc, en substance (et je me servirai ici d'une étiquette commode), d'un classicisme appliqué, une façon d'étendre à d'autres domaines une Altertumswissenschaft qui, au milieu du XIV siècle, n'avait pas encore un statut clair et autonome dans le système des connaissances et des praxis. A plusieurs reprises, Michele Feo a très justement mis en évidence "l'importance d'une lettre peu connue de la vieillesse, dans laquelle Pétrarque démontre que deux documents médiévaux, qui avaient été attribués à Jules César et à Néron, et qui devaient démontrer l'indépendance de l'Autriche par rapport à l'Empire, étaient des faux»; et il fournit la preuve de cette falsification en ayant recours à " des arguments stylistiques, historiques et linguistiques pour mettre le faussaire au pied du mur: anachronique est l'usage de la première personne du pluriel dans la bouche de l'autorité qui concède le privilège; non classique est le système de datation; erronée est l'attribution des titres d'Auguste et du titre de roi à César; absolument différent de celui des Anciens est le style 》.

Il s'agit assurément d'une page en or dans les annales de l'bumanisme. Mais, s'il m'est permis de mêler le plus et le moins, je dirais que ces lignes ne correspondent pas à une approche différente de celle que l'on retrouve dans bien des pages grises du De remediis, dans lesquelles Pétrarque utilise, à côté des chapitres consacrés aux grands enjeux moraux et religieux, la totalité de ses connaissances classiques pour passer en revue une multitude de situations bumaines, sans hésiter à prendre en considération les questions quotidiennes les plus banales, les realia les plus modestes, comme quand il cite Ovide, saint Augustin, l'Hortensius cicéronien, Virgile et je ne sais combien d'autres 
encore dans les vingt-cinq lignes d'un dialogue sur les dindons, les poulets, les poules, les abeilles et les pigeons ("De pavonibus, pullis, gallinis, apibus et columbis»).

Dans la perspective de la longue durée, il est évident que cette évolution pétrarquiste d'un classicisme pur et autosuffisant vers un classicisme pratique allait dans le sens de l'histoire. Dans son splendide prologue aux Elegantiae, Valla allait être catégorique. La langue de Rome a contribué d'une manière décisive au bien de l'bumanité, en formant les peuples aux arts libéraux, en leur donnant les meilleures lois, en leur permettant l'accès « ad omnem sapientiam» et en les sauvant de la barbarie. Toutes les disciplines dignes de l'homme libre s'expriment en latin et quand celui-ci fleurit, toutes les disciplines fleurissent aussi, alors qu'elles s'étiolent quand le latin faiblit. De nos jours, se lamente Valla, après de nombreux siècles pendant lesquels personne n'a parlé ni compris le latin, la philosophie, le droit et toutes les matières qu'avaient illustrées les anciens se retrouvent affaiblies et prostrées. Mais déjà pointe une nouvelle époque : la peinture, la sculpture, l'architecture qui étaient tombées en déchéance "cum litteris » ressuscitent peu à peu; et avec quelques efforts supplémentaires, conclut-il, on rétablira la langue de Rome, " et cum ea disciplinas omnes ». C'est une renaissance, la Renaissance.

Vers I440, Valla avait raison: ce qu'il fallait d'abord, c'était la maîtrise de la langue et de la littérature, de la philologie et de l'bistoire classiques, et de là allait surgir toute une civilisation profondément nouvelle. Un siècle après, ou plutôt deux, c'était un rêve qui s'était déjà évanoui: la conception de l'bumaniste comme grammaticus, la définition de l'bumanisme comme retour à l'Antiquité et pratique de la philologie, qui avaient fécondé toute la culture pendant des siècles et qui en étaient le fondement même avaient perdu leur caractère «impérialiste », "annexionniste » revendiqué à l'époque de Pétrarque et de Valla; l'Altertumwissenschaft n'était plus le moteur de la culture, mais une discipline auxiliaire de l'bistoire et de la littérature, dans un système où les sciences et les arts s'articulaient selon des critères différents des studia humanitatis. L'bumanisme avait démontré en particulier son incapacité constitutive à produire en latin une grande littérature de création, forte de possibilités d'avenir: l'innovation et même la révolution - surtout dans les genres réalistes, dans le théâtre, dans le roman -, étaient désormais l'affaire des langues vulgaires.

Eh bien Pétrarque était très conscient du fait que l'austère classicisme de sa jeunesse était insuffisant et insatisfaisant, autant d'un point de vue personnel que dans une perspective générale. Et même pour le proposer comme point de départ, il fallait en démontrer la validité en tant qu'objectif, soit en mettant à nu la fausseté d'un document qui se prétendait ancien, soit en convoquant tout le Parnasse pour disputer « de pavonibus, pullis, gallinis ... ». C'est là, comme 
je le disais, le grand projet de sa maturité : faire passer la sève classique de ses origines antiques à des terrains apparemment très lointains.

Il faut pourtant ajouter que cette évolution des méthodes et des objectifs constituait également une bonne opération pour acquérir les faveurs d'un public plus nombreux et prêt à recevoir de manière plus directe et attractive les enseignements de Pétrarque. Il ne s'agissait pas uniquement de rassembler des lecteurs riches et influents : un public aussi bigarré qu'abondant lui procurait autorité et prestige, et convertissait notre "philosophus » en " un bon investissement», une personnalité dont la présence auprès des Carrara et des Visconti valait son pesant d'or. Et Francesco savait qu'il ne pouvait obtenir qu'auprès de grands seigneurs de ce genre les conditions de vie et de travail qu'il désirait pour finir ses jours dans la paix et l'otium litteratum.

Il n'est pas superflu à cet égard de rappeler que Pétrarque menait un train de vie très cô̂teux et que, s'il avait fait autrement, il ne serait pas le Pétrarque que nous connaissons, l'homme qui marque de son empreinte toute la Renaissance. Dans la culture de son temps, un Francesco Fiorentino chanoine de Padoue, précepteur à Pise ou notaire à Vérone n'aurait pu aller beaucoup plus loin qu'un Rinaldo Cavalchini ou un Albertino Mussato, même en les surpassant de très loin par son talent, son esprit pénétrant et ses dons d'écrivain. Pétrarque ne serait jamais parvenu à l'exceptionnelle formation classique qui le caractérise s'il n'avait vécu à cheval entre l'Italie et la France, en effectuant de fréquents voyages, et s'il n'avait entretenu des relations suivies avec des personnalités qu'il n'aurait jamais rencontrées s'il était resté au fond de sa province. Assembler une bibliothèque comme la sienne, une bibliothèque dont on ne trouve pas d'égal non seulement parmi ses contemporains mais durant des siècles, et qui est de fait, et d'une manière très concrète, le véritable noyau de toute une civilisation, requérait des recherches, des déplacements, des copies, des soins et, surtout, du temps, beaucoup de temps disponible. Mais je ne sais pas si nous avons toujours suffisamment conscience qu'à l'époque de Pétrarque, pour mener la vie qui lui a permis de réaliser son grand cuvre littéraire et culturel, il n'avait aucun autre moyen à sa portée que de s'assurer la protection des puissants. C'est en ce sens que j'ai toujours compris cette déclaration controversée mais à juste titre pleine de fierté de la pénultième des Seniles : "en apparence, j'étais avec les princes; en réalité, ce sont eux qui étaient avec moi », "Nomine ego cum principibus fui, re autem principes mecum fuerant ». Pour vivre, créer et avoir ses aises pour l'étude, il n'existait aucun autre moyen que la protection des princes, et Pétrarque le sentait comme une évidence de plus en plus pressante, au fur et à mesure qu'il approchait de la cinquantaine. Il n'est pas nécessaire de démontrer que l'approche de ce cap, dans sa vie, fut pour lui une période pénible et difficile : la décision, drastique et scandaleuse, de chercher dans le Milan des Visconti le "portus» de sa maturité en dit 
suffisamment long sur les incertitudes et les craintes concernant son avenir qui l'avaient assailli durant le lustre précédent. (Et il n'est guère possible d'aborder ici un autre sujet, moins évident mais, du point de vue artistique, bien plus intéressant: la manière dont l'écrivain, dans diverses auvres de cette étape, essaye de déguiser sa crise de la cinquantaine en la faisant remonter dix ans plus tôt: considérons seulement pour l'heure que si l'action du Secretum se déroule en 1343, alors qu'il allait sur ses quarante ans, Francesco arrivait en réalité à Milan à peine quelques semaines avant le jour où - selon sa façon de compter - il allait avoir cinquante ans.) Au vrai, s'ily a une donnée d'évidence chez le Pétrarque de cette époque, c'est bien la sensation qu'il éprouve d'être parvenu à un carrefour décisif, à un moment où les seules perspectives qui s'offrent à lui sont les «vite reliquias - "les résidus de la vie» - que restant tempora vite », " la seule chose qui reste pour l'avenir», et que l'âge et la proximité de la mort exigent sans hésitation un acte de volonté, pour tracer la ligne d'borizon de la vieillesse: en tranchant les liens anciens, en choisissant une résidence en Italie, en vivant comme un sage dans la tranquillité de l'esprit et l'étude des lettres.

Comme il est notoire, c'est cet état d'âme, ce continuel souci d'avenir qui explique l'incessant va-et-vient de Pétrarque entre la France et l'Italie et d'un coin à l'autre de la Péninsule, s'assurant ici des bénéfices, se demandant ailleurs s'il devait ou non accepter un poste d'importance dans la curie ou dans la biérarchie ecclésiastique et cultivant toujours la faveur des grands, chez qui il trouva en dernier ressort l'issue que l'on sait et qui fait que nous le retrouvons serein, à Milan, en I353. Mais je crois que l'on n'a pas souligné suffisamment le fait que cette crise du demi-siècle a eu une profonde incidence sur la forme et l'apparence de la totalité du corpus pétrarquiste: c'est alors, en effet, que l'bumaniste donne à son cuvre ses caractères essentiels, qu'il décide des genres, des livres et des manières qu'il adopte; c'est alors aussi qu'il entreprend les travaux auxquels il va consacrer le reste de sa vie et qu'il décide de ceux qu'il va laisser «en attente » ou «en suspens». C'est alors, en somme, qu'il donne à son cuvre, pour l'essentiel, sa configuration définitive.

Le tout se traduit par une activité fébrile, vertigineuse, entre I349 et les premiers mois de 1353, une activité qui ne se répétera peut-être jamais avec la même intensité et qui se décline en deux opérations fondamentales. La devise de l'une pourrait être la citation biblique rapportée à la fin du Secretum : "Colligite quae superaverunt fragmenta, ne pereant» ("ramassez. les morceaux qui restent, pour qu'on ne les perde pas»). L'autre apparaît sans équivoque à travers le trouble, la presse et l'impatience que ressent Pétrarque et qui le poussent à essayer de conclure, presque en désespoir de cause, les grands projets qui lui avaient valu les lauriers du couronnement. Quoi qu'il en 
soit, l'un et l'autre de ces deux engagements traduisent la façon d'agir d'un bomme que l'âge a poussé non seulement à dresser le bilan de sa vie, mais également à prendre de sérieuses décisions pour les années à venir, et qui, à ce moment essentiel de son existence, se prépare à sauver, de sa production passée, tout ce qui peut l'être, à ordonner l'ensemble et à fixer les règles auxquelles il espère pouvoir se tenir durant le temps d'activité qui lui reste encore.

Durant les quelques minutes dont je dispose encore, je ne peux présenter la situation que de manière télégraphique, mais il s'agit, heureusement, de faits suffisamment connus. Le I3 janvier I350, Pétrarque signe la première des Familiares, "Ad Socratum suum ». Dans les mois qui suivent, il rédige la préface des Epystole, et, si je ne me trompe, si les chercheurs qui ont accepté mes conclusions ne se trompent pas non plus, il compose aussi le sonnet qui ouvre les Rerum vulgarium fragmenta, le Voi ch'ascoltate qui aujourd'bui nous a servi de point départ. Le prologue des Familiares annonce la tâche que les deux autres textes commencent à concrétiser : la compilation de trois grandes collections de lettres en prose, de vers latins et de vers en langue vulgaire, respectivement.

D'un point de vue littéraire et culturel, ce sont là des décisions de grande portée, que j'ai étudiées avec attention en diverses occasions. Mais à présent il me semble intéressant d'en souligner l'aspect humain et personnel. On trouve dans l'expérience de nombreux auteurs des situations parallèles, profondément bumaines. Arrivé à un certain âge, un homme passionné d'une discipline à laquelle il a consacré toute sa vie constate qu'il a appris plus de choses et composé plus de scripta minora qu'il n'est parvenu à achever de grands projets. Et c'est alors qu'il se décide à utiliser lesdits scripta minora pour tenter de leur insuffler les connaissances, la forme cohérente, les objectifs qu'il réservait pour une large part aux grands projets. Bref, les compilations des scripta minora viennent compenser d'une certaine manière le sentiment de frustration qu'ont laissé les grands projets qui n'ont pas abouti. Les collections des Familiares, des Epystole, des Rerum vulgarium fragmenta, avec leurs retouches, leurs améliorations, leurs pièces complémentaires, compensent en particulier l'inachèvement de l'Africa et du De viris. Mais, une fois conçus en tant que livres, ils marquent, pour l'essentiel, la voie que Pétrarque suivra durant le reste de sa vie et dont il ne se départira pas.

Jusqu'à ce qu'il entreprenne l'A frica et le De viris illustribus, l'bumaniste n'avait composé que des pièces brèves et indépendantes. A notre connaissance, que des poésies en langue vulgaire et des épîtres latines en vers et en prose. Les Rerum memorandarum, avec la netteté de leur schéma cicéronien, l'axe solide du De vita solitaria, l'inspiration passionnée du De otio religioso ou l'enchaînement médité du Secretum marquent des progrès notoires sur un itinéraire idéal vers des cuvres unitaires, de construction accomplie et d'archi- 
tecture liée. Même si chacun des éléments de ces livres pouvait être isolé de façon entièrement libre, l'ensemble était orienté par un plan aussi clair que cohérent. Mais cet itinéraire idéal s'arrête tout à coup et presque entièrement, vers I350, comme conséquence de la crise du passage de la quarantaine à la cinquantaine. Dans ce contexte, et surtout durant son dernier séjour de deux ans en Provence, Pétrarque avait pensé concilier ses efforts pour conclure l'Africa et le De viris avec le travail de révision, de classement et de rédaction de textes complémentaires nécessaires à la mise en forme des Familiares et des compilations analogues. Bientôt, la préparation de ces compilations se révéla agréable et féconde, tandis que l'"ultime retouche 》, la "lima ultima» des cuvres majeures s'avérait au contraire difficile et improductive.

L'Africa, en particulier, devint pour lui un véritable cauchemar. Le fait que $l$ " «opus [...] immodico labore confectum» n'avance pas lui pesait; affligé, il rêvait de le terminer et de se libérer de cette obsession. Et, bien évidemment, il hésitait et il doutait. Il craignait d'être parvenu à la limite de ses capacités de création et il espérait corriger une fois pour toutes, puis laisser de côté sans plus, cette pesante Africa, afin de se concentrer sur des activités spirituellement plus enrichissantes, les activités propres du "philosophus » qui était désormais son idéal: "magisterio senectutis doctior ac melior fieri》.

Celui qui lit La revisione petrarchesca dell'Africa, le splendide livre de Vincenzo Fera, ne peut que s'étonner de l'insignifiance et de l'inanité des corrections que l'écrivain a apportées au texte original. Bien que ces corrections soient précieuses pour comprendre l'élaboration du poème dans ses moindres aspects, dans ses détails de style les plus délicats, elles ne nous disent rien, cependant, sur la manière dont Pétrarque envisageait de combler les graves lacunes qui subsistaient encore et toujours, et rien non plus sur l'éventualité d'une refonte, sur la possibilité de remplacer des passages ou des épisodes notoirement faibles, ou encore sur une articulation plus efficace à partir d'autres matériaux. L'écrivain semble même souvent paralysé: " attende syllabam », "attende infra», "attende Lucanum», nous dit-on. Mais que devait-il et que devons-nous attendere? Comment remédier aux déficiences que Francesco lui-même percevait comme telles? La plupart du temps, nous ne le savons pas et probablement que lui non plus ne le savait pas.

Les notes découvertes par notre collègue se sont ainsi accumulées petit à petit, probablement " a più riprese», en plusieurs étapes: certaines de ces notes sont clairement postérieures à 1358 ; plusieurs ont tout l'air d'avoir été produites avant 1364, année de publication des Epystole; mais «les témoignages les plus clairs sur le travail de systématisation anquel le poème était soumis» ( $V$. Fera) doivent correspondre surtout à la période qui va du printemps à l'automne I352, juste avant le départ pour Milan. Eh bien, il n'y a aucun indice concernant ces reprises qui prouverait qu'après I347 ou, peut-être même 
après 1349, Pétrarque ait fait autre chose qu'introduire ou simplement envisager des corrections minimes, des détails.

A mes yeux, nous nous trouvons face à un échec, un "fallimento 》. Dans la majeure partie du texte qui nous est parvenu, il ne s'agit peut-être pas d" "un échec sur le plan artistique ou culturel ». Mais dans l'ensemble, et sur le plan bumain, personnel, pour moi, il ne fait aucun doute que l'Africa fut réellement un "fallimento». "On sait que dans les dernières années de sa vie, quand on lui rappelé l'Africa, Pétrarque se troublait et pâlissait ». Qu'y avait-il derrière cette soudaine pâleur? "La conscience d'un problème urgent et encore actuel ", a répondu un excellent critique. Pour ma part, je crois plutôt qu'il se sentait honteux de ne pas avoir la capacité ni le courage de s'acquitter de la dette qu'il avait contractée envers le public et envers lui-même - la dette qui consistait à rédiger une épopée digne de rivaliser avec l'Enéide.

Il n'y a pas lieu de le lui reprocher. Ce sentiment de honte l'bonore, et nous prouve qu'il était conscient de ses limites, conscient que l'Africa n'était pas une tâche faite pour lui. Parce que, comme je le disais précédemment, l'objectivité qui définit le poème épique était le genre le moins adéquat pour un homme qui ressentait le besoin de se produire, de se raconter et de s'inventer lui-même à travers l'écriture, et parce que, par là-même, le génie de Pétrarque est ostensiblement lyrique, et non pas épique; c'est le génie des unités mineures, des filigranes, de la nuance dans le détail: le génie dont témoignent les ébauches des rimes en langue vulgaire et le dessein que l'on retrouve tout au long des notes données en marge de l'Africa.

En fait, comme nous l'avons déjà indiqué, l'bumaniste s'était toujours occupé de textes brefs et c'est seulement après cette première époque de travail sur l'Africa et le De viris, probablement encouragé par l'espoir que ces deux cuvres avaient éveillé en lui, qu'il s'essaya aux livres proprement dits, aux cuvres de grande extension, douées d'une structure soutenue et cohérente (et, même alors, il est bien évident que les Rerum memorandarum ou le De vita solitaria ne sont pas à proprement parler des exemples de construction unitaire). Durant la période d'anxiété qui va de l'année de la Peste aux derniers mois passés en Provence, alors qu'il écrivait avec l'angoissante sensation que son temps s'achevait, Pétrarque dut sentir aussi qu'avec les cuvres produites en retouchant, révisant et complétant des petits textes ayant leur propre autonomie, comme les lettres ou les Rerum vulgarium fragmenta, il était sur la bonne voie, alors qu'il achoppait sur les livres de plus grande envergure. Cette approche fragmentaire était le mode de composition qui s'adaptait le mieux à ses dons de créateur et à ses exigences de "philosophus » théorique et d'homme du monde pratique, à son subjectivisme radical, à son désir obsessionnel de saisir et de traduire en mots sa vie réelle, de recréer son autobiographie idéale, de réaliser sa névrose de perfectionnisme. 
Voilà pourquoi, à dire vrai, il n'abandonnera plus jamais son projet et n'essaiera même pas d'abandonner cette voie. "Venit post multos una serena dies " "vint enfin, après bien d'autres, un jour serein », disait un vers classique. Pour Pétrarque aussi, après le tourbillon qu'avait été son coming of age, son partage de midi, après la Peste, les hésitations, les doutes, les inquiétudes et les frustrations des dernières années à Avignon, les jours sereins furent aussi au rendez-vous. A son arrivée à Milan, il entama le De remediis conçu probablement en Provence, il continua les Familiares, le Canzoniere, les Triumphi. A l'ombre de Sant'Ambrogio, on plus tard encore, il se remit à travailler à des cuvres comme les Epystole, le Bucolicum carmen ou le De vita solitaria, dont la structure « ouverte » rendait facile et pratique leur amélioration, sans qu'il soit nécessaire de les modifier de fond en comble; il écrivit aussi de brillants opuscules polémiques, parfois de valeur durable, comme le De ignorantia, mais toujours nés en dernier ressort de circonstances accidentelles; il s'employa à compléter le De gestis Cesaris, avec les moyens déjà assurés et avec la plus vive intelligence; et surtout, il compila le merveilleux testament spirituel et humain que sont les Seniles. Mais, depuis son installation à Milan, il ne commença plus de nouveau livre au sens strict du mot. Il n'acheva pas non plus celui qui aurait dî être le livre de sa vie. A mon avis, il a bien fait.*

\section{Francisco Rico}

\footnotetext{
* Je veux remercier mes chers amis Julio Murillo et Michel Jeanneret pour la première et pour la dernière version française de cette conférence.
} 